\title{
Small World Neural Network Based on FPGA
}

\author{
Aiqing Zhao ${ }^{a}$, Huiyan $\mathrm{Li}^{\mathrm{b}}$, Yuliang Liu, Chunxiao Han and Yanqiu Che \\ ${ }^{1}$ Tianjin Key Laboratory of Information Sensing \& Intelligent Control, Tianjin University of \\ Technology and Education, Tianjin, 300222, China \\ ajason_zaq@126.com, ${ }^{\mathrm{b}}$ Ihy2740@126.com, ${ }^{\mathrm{c}}$ yqche@tju.edu.cn
}

\begin{abstract}
Keywords: Small World Network, Field Programmable Gate Array, Mirror Image Memory.
\end{abstract}
\begin{abstract}
Statistical analysis results have shown that small-world characteristics are widespread in the cerebral cortex neuron network, which has long been proven an effective method to investigate the brain anatomy and functions. From the perspective of hardware, we built the FHN neural network model with small-world connectivity using FPGA (Field Programmable Gate Array). As a result, we observe the properties of neuron membrane potential are in line with the small-world characteristic of FHN neuron network model when impressed sinusoidal current with different frequencies. By the aforementioned rules, this paper demonstrates the effectiveness, and may underlie the construction of cerebral cortex network in the future.
\end{abstract}

\section{Introduction}

In recent years, through the quantitative analysis of neural network topology, the scientists found that the nervous system is generally has the characteristics of small world structure. An important feature of biological neural networks is the contradiction of synaptic connections, they usually have short average path length and high clustering coefficient [1]. For functional networks in human brain is described by Chialvo, the shortest path length $\mathrm{L}=6.0$, clustering coefficient $\mathrm{C}=0.15$. If they are regarded as random networks, then $L=6.0$ and $C=0.00089$. The appearance of brain function network structure is so-called small world characteristic [2-3]. Statistical analysis showed that an animal's brain cortex neuron network generally has the characteristics of small world [4-5]. Anatomy experiments prove that the small world network is probably the most general method of organization brain nerve system. Humphries think brain stem reticular structure can be portrayed as neural network of small world structure characteristics [6]. Recently based on the experiment of functional magnetic resonance imaging studies also showed that cerebral cortex and across regions of cortex show the characteristics of small world [7]. The nervous system model of small-world topological structure showed a stronger signal transmission speed, computing capacity and synchronization performance [8]. At present, because of the small world network model supports both local and remote way of information processing, it is one of the most effective methods, by which people recognize and study the brain anatomical and functional network [9].

The classic method for the simulation of the neural dynamic is based on software, but because the software based on the von Neumann serial processing computer system architecture, thereby limiting computing efficiency and the scale of software simulation. The hardware circuits become increasingly popular due to its high calculation efficiency and the ability for practical application. In this paper, we adopt Altera corporation Stratix III series of high-end FPGA EP3SL150. Using the FitzHugh-Nagumo(FHN) model builds small world neural network. It may underlie the construction of cerebral cortex network in the future.

\section{FitzHugh-Nagumo model}

The motivation for the FitzHugh-Nagumo model was to isolate conceptually the essentially mathematical properties of excitation and propagation from the electrochemical properties of sodium and potassium ion flow [10]. The model consists of a voltage-like variable having cubic nonlinearity 
that allows regenerative self-excitation via a positive feedback, and a recover variable having a linear dynamics that provides a slower negative feedback.

The model is sometimes written in the abstract form:

$$
\begin{aligned}
& \varepsilon \dot{V}_{i}=V_{i}-V_{i}^{3} / 3-W_{i}+I_{i}^{s y n}+I_{i} \\
& \dot{W}_{i}=V_{i}+a-b_{i} W_{i}
\end{aligned}
$$

Here, the neuron indices are $i=1,2,3 \ldots N$ and $N$ is the total number of the neurons, which are assembled into an uncoupled parallel array $\mathrm{V}$ and $\mathrm{W}$ denote the membrane potential and recovery variable respectively of each neuron, where $\varepsilon$, a and $\mathrm{b}$ denote the neuron parameters. $I_{i, n}^{\text {syn }}$ is the synaptic current of the ith neuron.

\section{Small world neural network model}

In order to build the small world neural network, and can be used stochastic reconnection method which put forward by the Watts and Strogatz.

Firstly, we can construct a regularization neural network, which each neuron and the adjacent $\mathrm{k}$ neurons connected via synapses, the left and right sides of each have $\mathrm{k} / 2$. Secondly, Connect the regularization neural network in the synapse random reconnection, set the rewiring probability for $\mathrm{p}$, the synaptic connections at the end of the neurons remain unchanged, while the other end of the synaptic neuron to randomly selected a new neurons in the network. While maintaining network only up to a connection between any two neurons, and no neurons connected to itself or the emergence of isolated neurons.

According to the above rules, when $0<\mathrm{p}<1$, the neural network possess small world structure features, as shown in Figure 1.

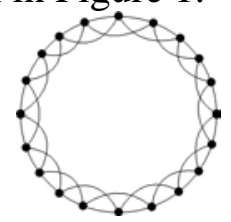

(a)

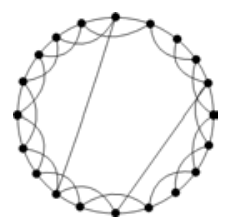

(b)

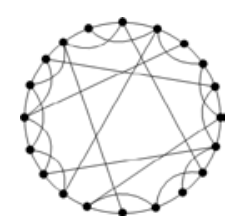

(c)

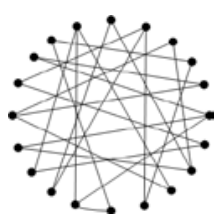

(d)

Figure 1. The network structure diagram. (a) Rules of the network, $\mathrm{p}=0$ (b) Small world network, $\mathrm{p}=0.05$ (c) Small world network, $\mathrm{p}=0.2$ (d) Random network, $\mathrm{p}=1$.

\section{The FPGA implementation of small world neural network model}

The small world neural network model is built as shown in Figure 2. The membrane voltage of FHN neural model through de-multiplexer block is stored in the mirror of the on-chip RAM. Through two lines of the Multiplexer block the membrane voltage participate in the synaptic current calculation of the structure. As shown in Fig. 1 of the construction of the small world information is stored in the GRAM. In the end, the synaptic current returns to the next FHN neuron model, which complete the signal transmission.

FHN parameterized model of the circuit principle as shown in Figure 3, Model consists of constant module, input and output module, the register module, addition and subtraction and multiplication module, etc. To realize network of sequential control, data operation steps of neural network calculation should be made. All neurons complete step calculation what is called a network status updates. In the network the n neurons use time-division multiplexing calculation method in turn. The structure of the electrical synapses is build, as shown in Figure 4.

In the network Neurons state information need to be stored up, to be involved in computation in different time periods. In a state of neurons information is read from the memory and the next neuron state information is written to memory, which two actions have been completed at the same time. 
However, because of the need to compute the synaptic current, depending on the network structure is likely to use any other neurons on the previous step of the membrane potential information. If the membrane potential of information has been updated, the calculation may be errors.

Therefore, in the membrane potential of information storage issues put forward the concept of image memory, as shown in Fig.5. Mirror image Memory is using two groups of storage store two step calculation values before and after the same variable. Respectively on two groups of memory read and write operations and Step calculation is finished, the swap position. The images storage structure is used, information updates will not lead to the original information are covered. After the completion of the calculation, the location of the data of simple swap also saved data replication operation time.

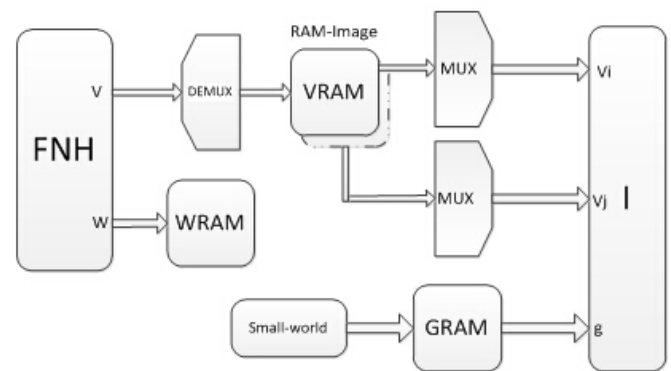

Figure 2. The small world neural network model

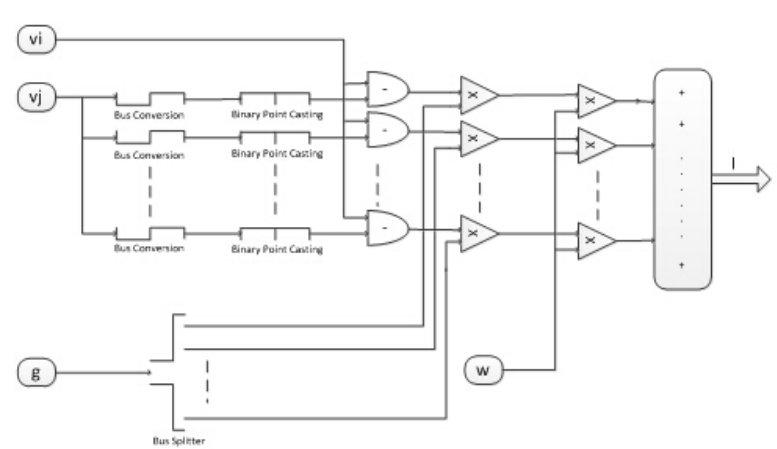

Figure 4 The electrical synapses

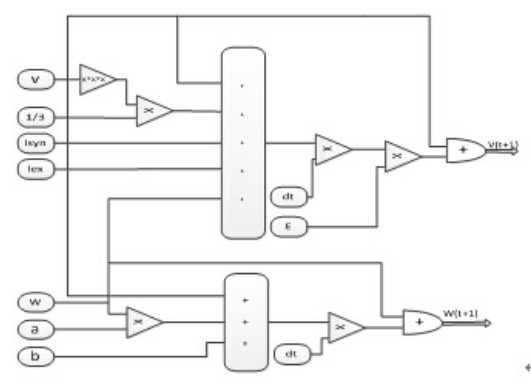

Figure 3. FHN parameterized model

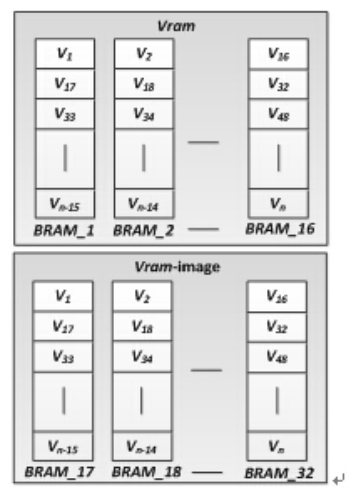

Figure 5 Mirror image memory of the membrane voltage

\section{Result and Analysis}

When the stimulus did not join sine wave, measurable FHN neuron network discharge cycle is 40 KHZ. Use of signal generator joins frequency from $10 \mathrm{KHZ}$ to $160 \mathrm{KHZ}$ and 70mA sine wave current stimulation. Through DA FHN neuron discharge can be observed on the oscilloscope waveform diagram as shown in the Figure 6. We can see FHN neuron discharge and sine wave resonance phenomenon, which accord with the characteristics of the small world FHN neural network.

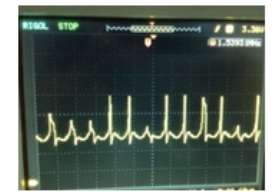

(a)

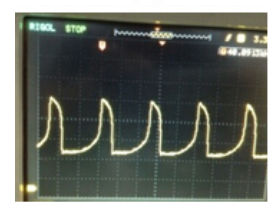

(d)

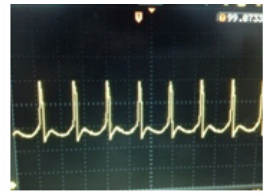

(b)

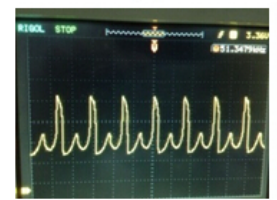

(e)

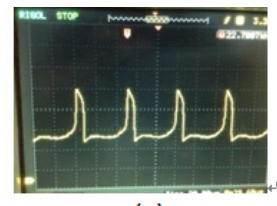

(c)

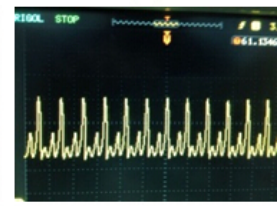

(f) 
Figure 6 (a) Join in 10khz sine wave current, the membrane voltage discharge (b) Join in 14khz sine wave current, the membrane voltage discharge (c) Join in $20 \mathrm{khz}$ sine wave current, the membrane voltage discharge (d) Join in 40khz sine wave current, the membrane voltage discharge (e) Join in $63 \mathrm{khz}$ sine wave current, the membrane voltage discharge (f) Join in 157khz sine wave current, the membrane voltage discharge.

\section{Conclusions}

Small world network is considered as one of the most effective methods to study brain anatomy and function networks. In this paper, we have implemented a small world network of FHN neurons by FPGA, which is of great significance to the future research of the characteristics of neural networks. FPGA's parallel computing ability is known as the most capable of achieving real meaning of artificial intelligence. It is similar to the signal transmission between neurons and may be able to effectively mimic the human brain in the future. And such as Parkinson, epilepsy mental disorders find the pathogenic mechanism. On the FPGA human can achieve the functional area of the neural network and replace diseased function area, to achieve the effect of the treatment of disease. Small world model is widespread in neural network. This paper set up the small world neural network which can provide a basis for its method.

\section{Acknowledgements}

This work was financially supported by the National Natural Science Foundation (61374182, 61401312) and the Tianjin Natural Science Foundation (13JCQNJC03700, 15JCYBJC19000). We would also acknowledge the support of Tianjin University of Technology and Education (Grants Nos: RC14-09, RC14-49 and RC14-59.

\section{References}

[1] E. Bullmore, O. Sporns, Complex brain networks: graph theoretical analysis of structural and functional systems, Nature Reviews Neuroscience, 2009, 10:186-198.

[2] D. R. Chialvo, Critical brain networks, Physica A, 2004, 340: 756-765.

[3] O. Sporns, D. R. Chialvo, M. Kaiser, et al., Organization, development and function of complex brain networks, Trends in Cognitive Sciences, 2004, 8:418-425.

[4] J. C. Reijneveld, S. C. Ponten, H. W. Berendse, et al., The application of graph theoretical analysis to complex networks in the brain, Clinical Neurophysiology, 2007, 118(11): 2317-2331.

[5] C. J. Stam, J. C. Reijneveld, Graph theoretical analysis of complex networks in the brain, Nonlinear Biomedical Physics, 2007, 1(1): 3.

[6] M. D. Humphries, K. Gurney, T. J. Prescott, The brainstem reticular formation is a small-world, not scale-free, network. Proceedings of the Royal Society (London) B, 2006, 273:503-511.

[7] R. Salvador, J. Suckling, M. R. Coleman, et al., Neurophysiological architecture of functional magnetic resonance images of human brain, Cerebral Cortex, 2005, 15:1332-1342.

[8] H. Hong, M. Y. Choi, B. J. Kim, Synchronization on small-world networks, Physical Review E, 2002, 65: 026139.

[9] C. H. Park, S. Y. Kim, Y. -H. Kim, et al., Comparison of the small-world topology between anatomical and functional connectivity in the human brain, Physica A, 2008, 387:5958-5962.

[10] D. J. Watts, S. H. Strogatz, Collective dynamics of 'small-world' networks, Nature, 1998, 393: 440-442. 